\title{
Progress in ultrasound lockin thermography
}

\author{
by A. Salerno', A. Dillenz ${ }^{2}$, D. Wu ${ }^{3}$, J. Rantala ${ }^{4}$ and G. Busse ${ }^{2}$ \\ ${ }^{1}$ ISPRA, CCR-TP 202, 21020 /spra (VA), Italia \\ ${ }^{2}$ Institut für Kunststoffpröfung und Kunststoffkunde (IKP), Universität Stuttgart, D-70569 Stuttgart, \\ Germany \\ ${ }^{3}$ Robert Bosch GmbH, D-73243 Wernau, Germany \\ ${ }^{4}$ Nokia Research Center, P.O. Box 407, FIN-00045 Nokia Group, Helsinki, Finland
}

\begin{abstract}
Ultrasound lockin thermography (ULT) is based on thermal wave imaging as a local response to periodical heat deposition. The substantial difference as compared to photothermal imaging and optical lockin thermography $(O L T)$ is that the heat source generating a thermal wave is provided by the defect itself due to the attenuation of amplitude modulated ultrasound. While friction effects heat areas with defects selectively, the slow amplitude modulation provides a periodicity of heating that results in the emission of long ranging thermal waves from defects. This way one has a dark field method to display defects in various materials even in the presence of complicated structures related with intact samples.
\end{abstract}

\section{Introduction}

New materials are increasingly being used in aviation and automobile industry. The development of health monitoring procedures for these applications includes methods of non-destructive testing. Important requirements are that the methods can be applied in a short time in a rough industrial environment and not only in the laboratory.

This is a strong argument against slow scanning systems like conventional ultrasonic inspection and also against methods involving safety regulations (e.g. laser radiation, $x$-rays...). A method that avoids these drawbacks is ultrasound lockin thermography (ULT) since it combines remote scanning with selective response to faults.

\section{Ultrasound lockin thermography}

The basic idea of optical lockin thermography (OLT) has been described previously [1-4]: Heat is deposited remotely by absorption of radiation. As the radiation is modulated, the generated temperature field oscillates. The local phase and amplitude are evaluated and presented as images derived from four primary images. Therefore such images display the local response of the sample to a modulated heat input.

Heat can also be generated inside the sample, e.g. by resistive heating or by loss angle heating [5-7]. The area of the hysteresis curve in the stress-strain diagram is the energy per volume converted into heat, hence the power density involves both the amplitude and the frequency. Therefrom it is obvious that higher frequencies require less amplitude of the mechanical vibration. That is why we use mechanical waves in the ultrasound range to generate heat.

Defects are areas of mechanical weakness (e.g. stress concentration, delamination, cracks) that may result in a locally enhanced hysteresis effect where either the loss angle is larger or the strain. In that case the defect reveals itself from its increased heat power density generated by conversion of ultrasound (Fig. 1a). When the amplitude of ultrasound is modulated, the local temperature is modulated in the same way so that the defect becomes a transmitter of a thermal wave (Fig. $1 \mathrm{~b}$ ) that is detected by lockin thermography at the surface of the inspected component (Fig. 1c) [8-9]. The resulting images display selectively areas of ultrasound absorption, which are mainly the defect areas. Due to this contrast mechanism, ULT images display the local imaginary part of modulus, while conventional ultrasonic techniques depend on the real part. Therefore this method is more efficient in 
detecting defective regions than overall heating used by OLT. Also it should be mentioned that in ULT the thermal wave is observed in transmission after it propagated from the defect site to the surface, while with OLT the thermal wave is reflected at the defect boundary. So the incoming and the reflected wave are superposed thereby causing interference effects and depth limitation [10-12].

\section{Experimental arrangement}

The experimental set-up we used is shown in fig. 2 . The amplitude modulated ultrasonic transducer (up to $1000 \mathrm{~W}$ at $20 \mathrm{kHz}$ ) heats the component periodically while the thermography system (AGEMA 900 Lockin) synchronised to the amplitude modulation acquires images in the wavelength range $8-12 \mu \mathrm{m}$ at a rate of 15 images/s. Due to the multiplex technique (where all sample points are monitored in parallel) we obtain images within three minutes even at modulation frequencies of $0.03 \mathrm{~Hz}$.

\section{Results}

Results are presented for metals and non-metals. A comparison with conventional lockin thermography (excitation with light) is performed.

\subsection{Metal samples}

In conventional applications one still uses metals whose failure mechanism is known. The main faults to be investigated are cracks and corrosion. This is of particular interest for the inspection of ageing aircraft in order to monitor their reliability.

\subsubsection{Vertical cracks}

Vertical cracks are hard to detect with photothermal methods and with OLT since the boundary is oriented along the direction of thermal wave propagation. However, fig. 3 shows that ULT can reveal vertical cracks since they are heated selectively by friction effects.

\subsubsection{Hidden corrosion in stringers}

The conventional lockin image shows two aluminium plates with corrosion between them. This image needs some interpretation since it is dominated by the intact structure of the rivets, while the ultrasound lockin image shows only the corroded areas (fig. 4).

\subsection{Nonmetal samples}

In order to obtain maximum strength at low weight one uses non-metal materials for aerospace applications. In such cases one is interested to detect areas of impact or delaminations.

\subsubsection{Impacts in CFRP}

The example of impact detection is intended to show the influence of a coupling medium between ultrasonic transducer and sample: With direct coupling we obtain a contrasted standing wave pattern in the phase image and lower intensity in the amplitude image (fig. 5). However, the use of a suitable coupling medium has the effect that standing waves are more efficiently attenuated so that the interference patterns are weaker and more elastic energy is injected into the sample due to better impedance matching. Therefore the defects stand out more clearly. 


\subsubsection{Coated wood}

Fig. 6 shows a plate of GFRP coated wood. The upper area of the sample has partly lost adhesion. The amplitude image shows this defect together with a standing wave pattern that displays some directionality effects. Such phenomena result in inhomogeneous heating as it is known from illumination in OLT. It is obvious that the phase image is not disturbed by this intensity distribution, an effect observed previously [13]. Hence the phase image shows the whole defective area.

\subsubsection{Delaminations in C-SiC}

In order to investigate the depth range of ULT we measured a $33 \mathrm{~mm}$ thick C-SiC sample (a material used for space vehicle re-entry) containing a delamination located $7 \mathrm{~mm}$ underneath the front surface. The results are shown in fig. 7 . The front surface image displays the delamination in the left upper corner. The back side image shows the delamination also, even beyond $26 \mathrm{~mm}$ of C-SiC. This indicates that depth range is presumably limited rather by noise than by thermal wave interference effects known in OLT [10-12].

\section{Conclusion}

Ultrasound lockin thermography (ULT) allows for selective detection of defects that are characterised by a locally enhanced hysteresis effect. Typical defects can be detected in materials such as metal, coated wood, and reinforced fibre composites (CFRP, GFRP and $\mathrm{C}-\mathrm{SiC}$ ). Different types of defects such as delaminations, impacts and even vertical cracks can be revealed this way. Problems to be investigated in future include

- improvement of acoustic input coupling,

- depth range and depth profiling,

- vertical and lateral resolution.

Comparison will also be made to the results obtained at IKP with ultrasonic pulse thermography where ultrasonic pulses (instead of amplitude modulation) are used to achieve selective heat pulse emission from defect areas.

The final goal of these investigations is the applicability of ultrasound stimulated thermography to larger samples in the field of quality control for rapid defect identification.

\section{REFERENCES}

[1] CARLOMAGNO (G. M.), BERARDI (P. G.) - Unsteady thermotopography in nondestructive testing, Proceedings of the 3rd Biannual Information Exchange, Aug. 2426, St. Louis/USA, 1976, p. 33-39.

[2] BEAUDOIN (J.-L.), MERIENNE (E.), DANJOUX (R.), EGEE (M.) - Numerical system for infrared scanners and application to the subsurface control of materials by photothermal radiometry, Infrared technology and applications, SPIE 590, 1985, p. 287-292.

[3] KUO (P.K.), FENG (Z.J.), AHMED (T.), FAVRO (L. D.), THOMAS (R. L.), HARTIKAINEN (J.) - Parallel thermal wave imaging using a vector lock-in video technique, Hess P., Pelzl J. (Eds.), Photoacoustic and photothermal phenomena, Springer-Verlag, Heidelberg, 1987, p. 415-418.

[4] BUSSE (G.), WU (D.), KARPEN (W.) - Thermal wave imaging with phase sensitive modulated thermography, J- Appl. Phys. 71, 1992, p. 3962-3965.

[5] SPATE, Patent No PCT/GB 79/00081 and DE 2952809 C2.

[6] HENNEKE (E. G.), REIFSNIDER (K. L.), and STINCHCOMB (W. W.) - Thermography:an NDI method for damage detection. Journal of Metals, 31, 1979, p. 11-14. 
[7] BUSSE (G.), BAUER (M.), RIPPEL (W.), and WU (D.) - Lockin vibrothermal inspection of polymer composites. QIRT'92, Editions Européennes Thermique et Industrie, Paris, 1992 p.154-159.

[8] RANTALA (J.), WU (D.), and BUSSE (G.) - Amplitude modulated lockin vibrothermography for NDE of polymers and composites. Research in Nondestructive Evaluation 7, 1996, p. 215-228.

[9] RANTALA (J.), WU (D.), and BUSSE (G.) - NDT of Polymer Materials Using Lock-in Thermography with Water-coupled Ultrasonic Excitation. NDT\&E International 31, 1998, p. 43-49.

[10] BUSSE (G.), Optoacoustic phase angle measurement for probing a metal, Appl. Phys. Lett. 35, 1979, p. 759-760.

[11] THOMAS (R. L.), POUCH (J. J.), WONG (Y. H), FAVRO (L. D.), KUO (P. K.), ROSENCWAIG (A.) - Subsurface flaw detection in metals by photoacoustic microscopy, J. Appl. Phys. 51, 1980, p. 1152-1156.

[12] LEHTO (A.), JAARINEN (J.), TIUSANEN (T.), JOKINEN (M.), LUUKKALA (M.) -, Magnitude and phase in thermal wave imaging, Electr. Lett., 17, 1981, p. 364-365.

[13] ROSENCWAIG (A.), BUSSE (G.) - High resolution photoacoustic thermal wave microscopy, Appl. Phys. Lett., 36,1980, p. $725-727$.

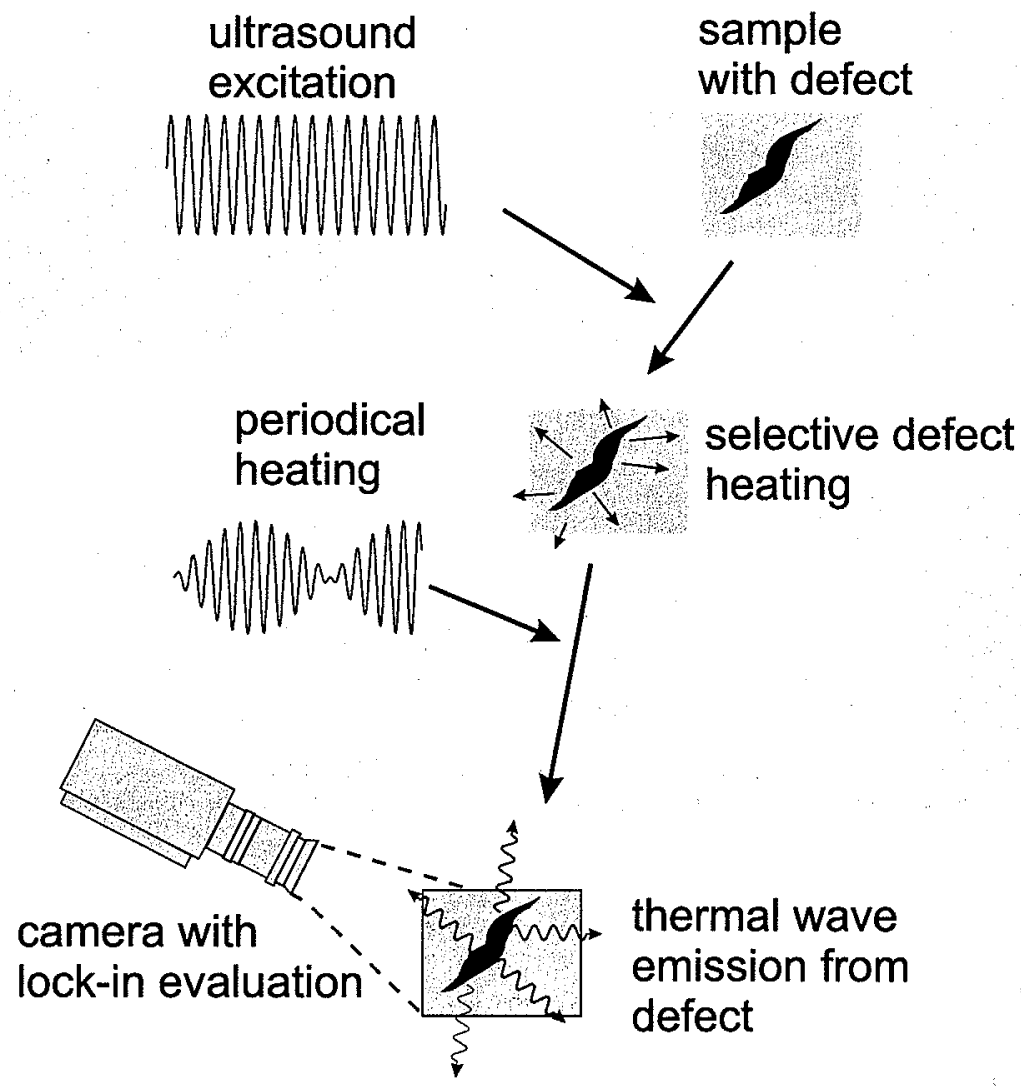

Fig. 1. Use of modulated ultrasound heating for local excitation of thermal waves 
http://dx.doi.org/10.21611/qirt.1998.024

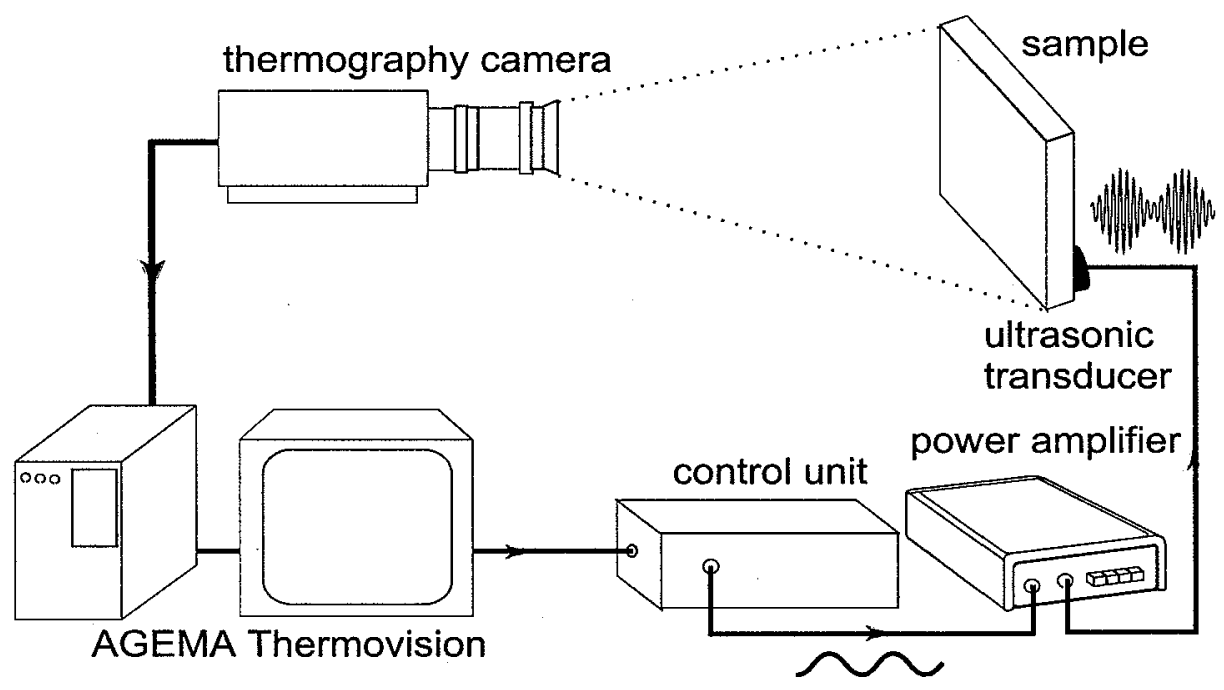

Fig. 2. Schematical diagram of ultrasound lockin thermography (ULT)
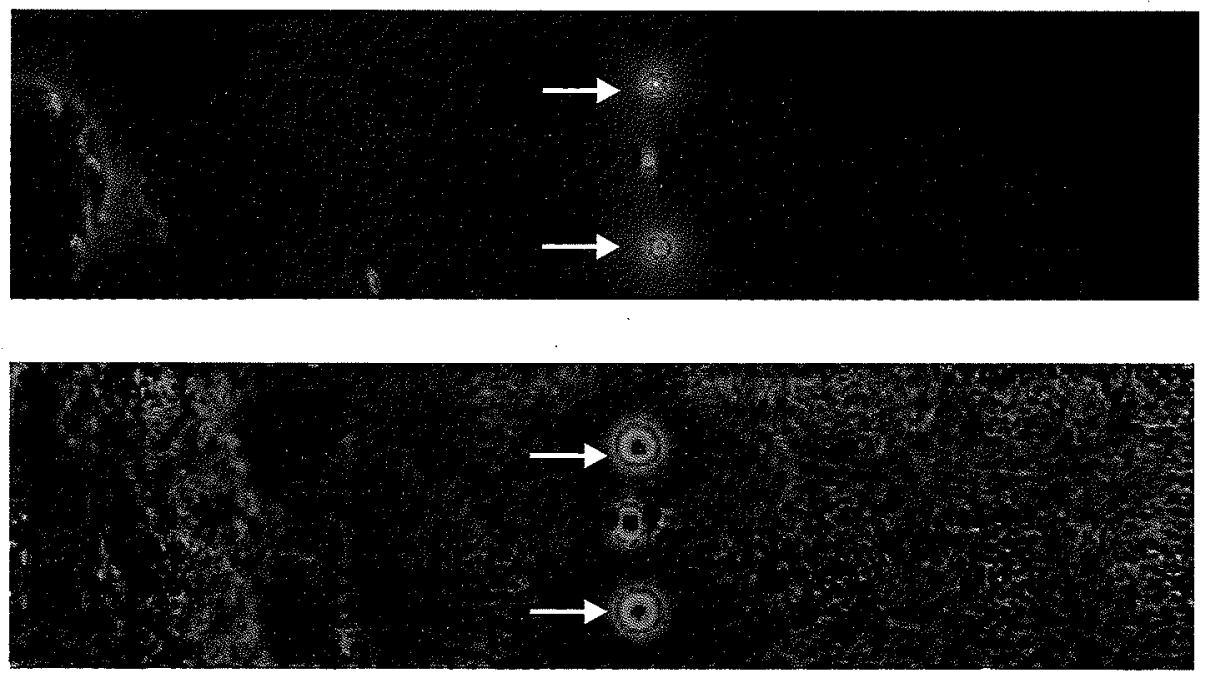

Fig. 3. Detection of a vertical crack in steel with ULT (Lockin frequency: $0.23 \mathrm{~Hz}$. US power: $300 \mathrm{~W}$ ). Top: amplitude. Bottom: phase. Crack tips are indicated by arrows. 
http://dx.doi.org/10.21611/qirt.1998.024
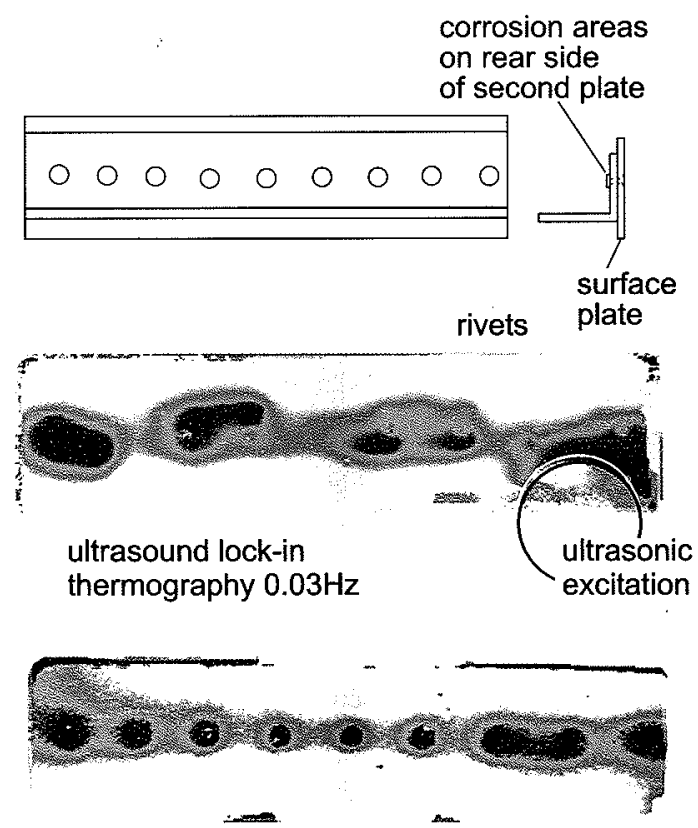

lockin-thermography $0.03 \mathrm{~Hz}$

Fig. 4: Detection of corrosion in stringers. Top: sample geometry.

Middle: ultrasound excitation.

Bottom: conventional lockin with light excitation
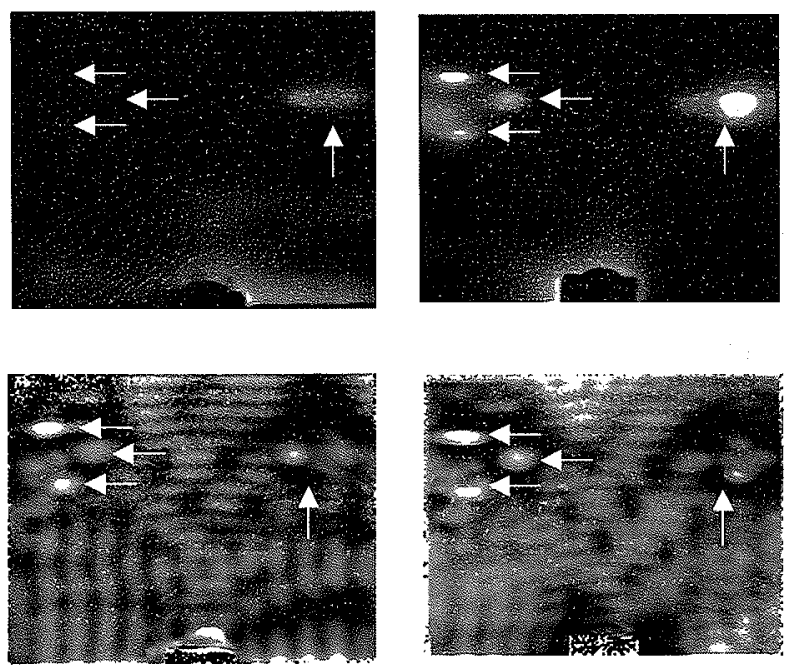

Fig. 5: Detection of 4 impacts (see arrows) in CFRP plate. Top: amplitude. Bottom: phase. The left set of images was produced by attaching the transducer directly to the sample. The right images show the effect of a coupling medium between the transducer and the sample. 
http://dx.doi.org/10.21611/qirt.1998.024

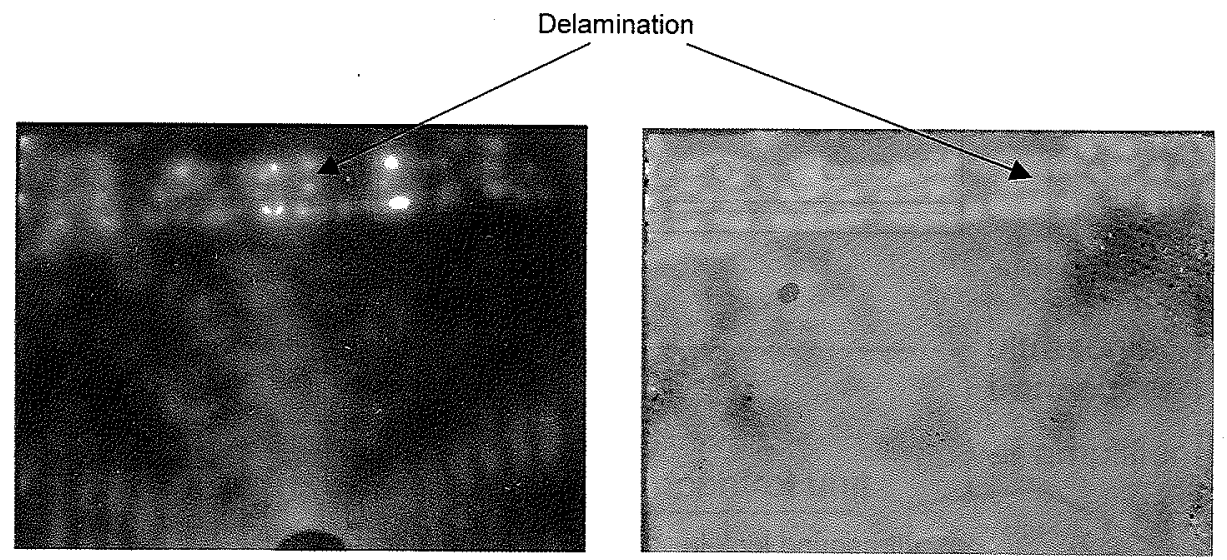

Fig. 6. Delamination in a GFRP coated wooden sample. Left: amplitude. Right: phase. Lockin frequency: $0.03 \mathrm{~Hz}$. US power: $200 \mathrm{~W}$.

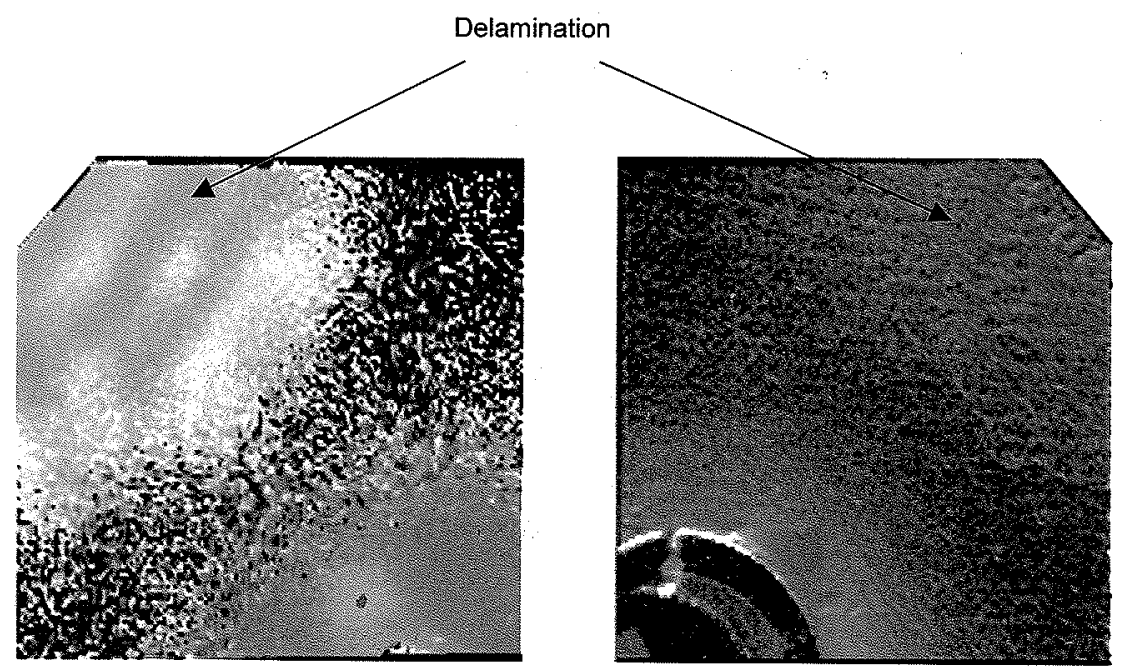

Fig. 7. Left: C-SiC with delamination in the upper left corner (US power: $500 \mathrm{~W}$, frequency: $0.03 \mathrm{~Hz}$ ). Right: Same sample from the rear side (frequency: $0.02 \mathrm{~Hz}$ ). 